\title{
Mineral Contents of Sri Lankan Rice Varieties as Affected by Inorganic Fertilization
}

\author{
H.M.A.J. Herath, G.A.P. Chandrasekara ${ }^{1 *}$, U. Pulenthiraj ${ }^{1}$, C.M.N.R. Chandrasekara ${ }^{2}$ \\ and D.G.N.G. Wijesinghe ${ }^{3}$
}

\author{
Postgraduate Institute of Agriculture \\ University of Peradeniya \\ Sri Lanka
}

\begin{abstract}
Application of inorganic fertilizers may incorporate minerals into rice grains. Distribution of minerals in rice grains vary in bran and kernel. The aim of the present study was to compare mineral contents (MCs) of bran and kernels of selected newly improved rice varieties in Sri Lanka with and without fertilizers. Twenty rice varieties were tested. Rice bran and rice kernels were analyzed for $\mathrm{Ca}, \mathrm{Mg}, \mathrm{Mn}$ and $\mathrm{Zn}$ using Atomic Absorption Spectrophotometer. Calcium contents of brans and kernels ranged from 952 to $1605 \mathrm{mg} / \mathrm{kg}$ and 613 to $1107 \mathrm{mg} / \mathrm{kg}$ dry matter in fertilized varieties, respectively. High MCs were observed in fertilizer applied varieties. Higher MCs were found in the bran of rice grains. The MCs of rice grains were significantly different among the varieties and affected by fertilizer application and processing. Applications of inorganic fertilizers strengthened the MCs of rice kernels and bran.
\end{abstract}

Keywords: Bran, fertilizer, kernel, mineral contents, rice

\section{INTRODUCTION}

Cereals are the edible grains of Gramineae family. There are a variety of cereals including rice, wheat, rye, oats, barley, maize, millet and sorghum. Rice is the staple food for more than half of the worlds' population being the second most leading cereal next to wheat worldwide(Anjum et al., 2007). Rice grain provides $75-80 \%$ of starch, $12 \%$ water, $7 \%$ of protein, fats, B vitamins mainly thiamine, riboflavin and niacin and minerals such as calcium, magnesium, phosphorus, manganese, copper, and iron (Oko et al., 2012). The prominent cultivating species of rice in Sri Lanka is Oryza sativa.

Minerals are essential nutrients for human growth and development. They play a vital role in the effective functioning of the human systems. $\mathrm{Ca}$ and $\mathrm{Mg}$ are known as major minerals which require $>100 \mathrm{mg} /$ day for the body functions and $\mathrm{Zn}$ and $\mathrm{Mn}$ are known as trace minerals which require $<100 \mathrm{mg} / \mathrm{day}$. One of the major reasons for the loss of essential micronutrients from rice is the high polishing rate (Abbas et al., 2011).

\footnotetext{
Department of Applied Nutrition, Wayamba University of Sri Lanka, Sri Lanka

Department of Agriculture, Sri Lanka School of Agriculture, Kundasale, Sri Lanka

Department of Food Science and Technology, Faculty of Agriculture, University of Peradeniya, Sri Lanka

* Corresponding author: anomapriyan@yahoo.com
} 
Department of Agriculture has introduced newly developed rice varieties having higher yield potential, pest and disease resistance, response to fertilizers and better grain quality. The growing environment has a great influence on the composition of the rice grain. (Abbas et al., 2011). Urea, Triple Super Phosphate and Muriate of potash are the three key chemical fertilizers used in Sri Lanka (Ekanayake, 2009). These chemical fertilizers commonly consist of three major components, namely as nitrogen, phosphorus and potassium. The aim of the current study was to determine the impact of the application of fertilizers on the mineral contents of bran and kernel fractions of newly improved rice varieties in Sri Lanka.

\section{Sample preparation}

\section{METHODOLOGY}

Random sampling method was used to obtain the rice grain samples from rice fields in the Rice Research and Development Institute in Bathalagoda, Rice Research Station. Twenty inorganic fertilized (Urea, Mureate of Pottash and Triple super Phosphate in 225:60:55 $\mathrm{kg} / \mathrm{ha}$, respectively) and non-fertilized Sri Lankan rice varieties, At 353, At 362, At 303, H4, Bw 276 - 6B, Ld 368, Bg 450, Bg 400 - 1, Bg 360, Bg 94 - 1, Bg 379 - 2, Bg 300, Bg 305, Bg 357, $B w$ 367, $B w$ 451, Ld 371, At 306, At 309 and At 405were obtained. Three representative samples from each variety were obtained. Rice samples were dehusked using a rice milling machine (Rice machine, Satake Engineering Co Ltd, Japan). The whole grains were polished (up to 90\%) with a rice miller (Rice husker and polisher PM 500, Satake Engineering Co Ltd, Japan). Milling and polishing processes were performed at the Institute of Postharvest Technology of Sri Lanka, Anuradhapura. Rice grains and bran were separately collected. Polished raw rice grains were finely ground using a grinder (Phillips HR 2011, Koninklijke Phillips Electronics N.V., China). The ground samples were passed through a sieve with the mesh size of $1 \mathrm{~mm}$. Rice grains and counterpart bran samples were oven dried at $105^{\circ} \mathrm{C}$ for constant weight to remove moisture. All the samples were stored in freezer (DW-86L626 Haier, U.K.) at $-80^{\circ} \mathrm{C}$ until further analysis.

\section{Determination of mineral contents}

A $0.5 \mathrm{~g}$ sample was measured into microwave digestion vessel using a top loading balance (Adventurer ${ }^{\mathrm{TM}}$ OHAUS, U.S.A.) followed by addition of $2 \mathrm{ml}$ of concentrated $\mathrm{HCl}(35 \%)$ and $2 \mathrm{ml}$ of concentrated $\mathrm{HNO}_{3}(69 \%)$. The mixture was allowed for predigesting and digested for one hour using microwave digestion system (MARS 6 One touch technology CEM Corporation, North Carolina). The digested samples were filtered and volume up to $50 \mathrm{ml}$ using deionized water. Mineral contents were determined using atomic absorption spectrophotometry. A series of standards for selected minerals were prepared from the standard stock solutions $(1000 \mathrm{mg} / \mathrm{l})$ of corresponding minerals as $1 \mathrm{mg} / \mathrm{l}, 2 \mathrm{mg} / \mathrm{land} 3 \mathrm{mg} / \mathrm{l}$. The mineral contents of the standards and the samples were measured using atomic absorption spectrophotometer (iCETM 3000 series Thermo Scientific, USA). The mineral contents were calculated on drymatter basis. All the samples were analyzed in triplicates.

\section{Statistical Analysis}

The differences of mean values among kernels and brans of fertilized and non fertilized treatments were determined using multivariate analysis of variance (MANOVA) followed by Tukey's Honestly Significant Differences (HSD) multiple rank test at $p \leq 0.05$ significance level. SPSS version (16.0) was used for the statistical analysis. 


\section{RESULTS AND DISCUSSION}

Tables 1 to 3 present $\mathrm{Ca}, \mathrm{Mg}, \mathrm{Zn}$ and $\mathrm{Mn}$ contents of rice varieties constituted of red and white pericarps. In general, higher $\mathrm{Ca}, \mathrm{Mg}, \mathrm{Zn}$ and $\mathrm{Mn}$ contents were observed in bran than the kernel for all rice varieties with red pericarp (Table 1). Further, it was noted that $\mathrm{Ca}, \mathrm{Mg}, \mathrm{Zn}$ and $\mathrm{Mn}$ contents of fertilized brans and kernels were higher than those of corresponding non-fertilized rice samples.

\section{Ca content of rice varieties}

The bran fraction of fertilizer added rice showed a range of Ca contents varying from 1368 to $1911 \mathrm{mg} / \mathrm{kg}$ (Table 1). The fertilized kernel fractions had Ca contents varying from 613 to $1107 \mathrm{mg} / \mathrm{kg}$ of rice varieties with red pericarp. The variety BG 305 reported the highest content of Ca for fertilized bran and kernels whereas BW 451 and AT 309 had the highest contents of $\mathrm{Ca}$ of non- fertilized bran and kernels, respectively.

\section{Mg content of rice varieties}

The Mg contents of fertilized and non- fertilized rice kernels varied 224-655 and 237- 452 $\mathrm{mg} / \mathrm{kg}$, respectively. Non-fertilized kernels of AT 362, AT 303 and H4 red rice varieties showed higher $\mathrm{Mg}$ content than those of fertilized counterparts. The range of $\mathrm{Mg}$ contents of rice bran and kernel with white pericarp ranged from 1230 to 1068 and from 487 to $212 \mathrm{mg} / \mathrm{kg}$ of the bran and kernel, respectively.

\section{$\mathrm{Zn}$ content of rice varieties}

Fertilized and non-fertilized brans of rice with red pericarp had a Zn content ranged from 121192 and 123-163 mg/kg, respectively. In general, fertilized and non-fertilized kernels of rice with red pericarp showed a $\mathrm{Zn}$ content ranged from 15 to $26 \mathrm{mg} / \mathrm{kg}$. The $\mathrm{Zn}$ contents of kernels were 6-10 times lesser than that of bran of rice with red pericarp. The $\mathrm{Zn}$ and $\mathrm{Mn}$ contents of the rice varieties were comparatively lower than the $\mathrm{Ca}$ and $\mathrm{Mg}$ contents (Table 3). Among non-fertilized rice brans AT 309 had the highest $(210.5 \mathrm{mg} / \mathrm{kg})$ and BG 360 had the lowest $(106.7 \mathrm{mg} / \mathrm{kg}$ ) zinc contents. The rice varieties except AT 309, AT 306, BG 300 and BG 3792 , explicated significantly higher $\mathrm{Zn}$ contents in fertilized brans compared to those of nonfertilized $(P<0.05)$.

\section{Mn content of rice varieties}

Among rice varieties with red pericarp LD 368 reported the highest content of Mn of fertilized and non-fertilized rice bran whereas BW 276-6B had the highest content of Mn of kernels (Table 1). The Mn contents of the rice varieties with white pericarp were comparatively lower than those of $\mathrm{Ca}$ and $\mathrm{Mg}$. Among fertilized bran, Mn contents varied from 214 to $131 \mathrm{mg} / \mathrm{kg}$. Among non-fertilized rice brans BG 450 had the highest Mn content (Table 3). 
Table 1. Mean calcium, magnesium, zinc and manganese contents of rice varieties with red pericarp $(\mathrm{mg} / \mathrm{kg})$

\begin{tabular}{|c|c|c|c|c|}
\hline & \multicolumn{4}{|c|}{ Calcium } \\
\hline & \multicolumn{2}{|c|}{ Fertilized } & \multicolumn{2}{|c|}{ Non-fertilized } \\
\hline & Bran & Kernel & Bran & Kernel \\
\hline AT 353 & $1368.4 \pm 29.3^{\mathrm{a} 1}$ & $682.5 \pm 6.6^{\mathrm{b}^{*}}$ & $904.9 \pm 15.5^{\mathrm{c} 2}$ & $545.6 \pm 8.2^{\mathrm{d} \#}$ \\
\hline AT 362 & $1428.6 \pm 8.2^{\mathrm{a} 1}$ & $696.4 \pm 7.7^{\mathrm{b}^{*}}$ & $903.8 \pm 4.5^{\mathrm{c} 2}$ & $520.7 \pm 7.4^{\mathrm{d \#}}$ \\
\hline AT 303 & $1604.9 \pm 17.1^{\mathrm{a} 1}$ & $613.4 \pm 12.5^{b^{*}}$ & $749.5 \pm 12.2^{\mathrm{c} 2}$ & $690.9 \pm 17.1^{\mathrm{c \#}}$ \\
\hline H4 & $1571.6 \pm 31.5^{\mathrm{a} 1}$ & $616.4 \pm 4.6^{\mathrm{b}^{*}}$ & $689.1 \pm 13.7^{\mathrm{c} 2}$ & $589.6 \pm 8.2^{\mathrm{c \#}}$ \\
\hline BW 276-6B & $1486.3 \pm 14.5^{\mathrm{a} 1}$ & $1107.2 \pm 11.7^{\mathrm{b}^{*}}$ & $671.7 \pm 2.2^{\mathrm{c} 2}$ & $561.7 \pm 6.8^{\mathrm{c \#}}$ \\
\hline \multirow[t]{2}{*}{ LD 368} & $1911.1 \pm 2.7^{\mathrm{a} 1}$ & $730.1 \pm 4.4^{b^{*}}$ & $836.2 \pm 18.0^{\mathrm{c} 2}$ & $560.0 \pm 6.2^{\mathrm{d} \#}$ \\
\hline & \multicolumn{4}{|c|}{ Magnesium } \\
\hline AT 353 & $1208.5 \pm 6.8^{\mathrm{a} 1}$ & $279.2 \pm 0.4^{\mathrm{b}^{*}}$ & $1157.5 \pm 6.3^{\mathrm{c} 2}$ & $242.2 \pm 1.2^{\mathrm{d} \#}$ \\
\hline AT 362 & $1206.5 \pm 0.6^{\mathrm{a} 1}$ & $224.4 \pm 2.6^{b^{*}}$ & $1175.3 \pm 6.5^{\mathrm{c} 2}$ & $271.9 \pm 1.8^{\mathrm{d} \#}$ \\
\hline AT 303 & $1203.8 \pm 12.5^{\mathrm{a} 1}$ & $240.8 \pm 2.1^{\mathrm{b}^{*}}$ & $1112.9 \pm 6.2^{\mathrm{c} 2}$ & $287.6 \pm 1.0^{\mathrm{d} \#}$ \\
\hline H4 & $1181.6 \pm 6.0^{\mathrm{a} 1}$ & $223.8 \pm 0.6^{b^{*}}$ & $1117.4 \pm 11.4^{\mathrm{c} 2}$ & $237.0 \pm 1.6^{\mathrm{d} \#}$ \\
\hline BW 276-6B & $1176.9 \pm 13.0^{\mathrm{a} 1}$ & $655.0 \pm 6.7^{b^{*}}$ & $1094.2 \pm 10.5^{\mathrm{c} 2}$ & $452.3 \pm 2.8^{\mathrm{d} \#}$ \\
\hline \multirow[t]{2}{*}{ LD 368} & $1204.5 \pm 6.6^{\mathrm{a} 1}$ & $410.1 \pm 1.4^{b^{*}}$ & $1162.2 \pm 11.5^{\mathrm{c} 2}$ & $344.5 \pm 2.2^{\mathrm{d \#}}$ \\
\hline & \multicolumn{4}{|c|}{ Zinc } \\
\hline AT 353 & $127.5 \pm 1.2^{\mathrm{a} 1}$ & $17.5 \pm 0.4^{\mathrm{b}^{*}}$ & $153.5 \pm 0.7^{\mathrm{c}^{*}}$ & $16.1 \pm 0.1^{\mathrm{d} \#}$ \\
\hline AT 362 & $192.2 \pm 1.6^{\mathrm{a} 1}$ & $21.5 \pm 0.2^{b^{*}}$ & $152.0 \pm 1.8^{\mathrm{c}^{*}}$ & $18.5 \pm 0.3^{\mathrm{d} \#}$ \\
\hline AT 303 & $121.1 \pm 1.9^{\mathrm{a} 1}$ & $15.1 \pm 0.4^{b^{*}}$ & $163.1 \pm 1.3^{\mathrm{c}^{*}}$ & $24.6 \pm 0.2^{\mathrm{d} \#}$ \\
\hline H4 & $188.1 \pm 1.4^{\mathrm{a} 1}$ & $24.8 \pm 0.2^{\mathrm{b}^{*}}$ & $128.7 \pm 1.0^{\mathrm{c}^{*}}$ & $22.9 \pm 0.3^{\mathrm{d} \#}$ \\
\hline BW 276-6B & $144.3 \pm 0.8^{\mathrm{a} 1}$ & $23.5 \pm 0.2^{\mathrm{b}^{*}}$ & $122.8 \pm 1.0^{\mathrm{c}^{*}}$ & $26.4 \pm 0.2^{\mathrm{d} \#}$ \\
\hline \multirow[t]{2}{*}{ LD 368} & $154.7 \pm 0.6^{\mathrm{a} 1}$ & $18.4 \pm 0.1^{b^{*}}$ & $130.3 \pm 0.7^{\mathrm{c}^{*}}$ & $22.4 \pm 0.4^{\mathrm{d} \#}$ \\
\hline & \multicolumn{4}{|c|}{$\begin{array}{c}\text { Manganese } \\
\end{array}$} \\
\hline AT 353 & $122.0 \pm 1.6^{\mathrm{a} 1}$ & $20.6 \pm 0.8^{\mathrm{b}^{*}}$ & $99.5 \pm 2.2^{\mathrm{a} 1}$ & $16.8 \pm 0.4^{\mathrm{cH}}$ \\
\hline AT 362 & $157.5 \pm 1.5^{\mathrm{a} 1}$ & $22.1 \pm 1.2^{\mathrm{b}^{*}}$ & $120.6 \pm 1.3^{\mathrm{c} 2}$ & $18.7 \pm 0.8^{\mathrm{d} \#}$ \\
\hline AT 303 & $126.1 \pm 0.7^{\mathrm{a} 1}$ & $20.0 \pm 0.8^{\mathrm{b}^{*}}$ & $106.3 \pm 2.3^{\mathrm{a} 1}$ & $19.8 \pm 1.7^{\mathrm{c \#}}$ \\
\hline H4 & $125.9 \pm 0.6^{\mathrm{a} 1}$ & $21.7 \pm 1.2^{\mathrm{b}^{*}}$ & $106.6 \pm 1.5^{\mathrm{a} 1}$ & $21.5 \pm 1.4^{\mathrm{c \#}}$ \\
\hline BW 276-6B & $203.2 \pm 2.5^{\mathrm{a} 1}$ & $29.2 \pm 0.6^{\mathrm{b}^{*}}$ & $121.8 \pm 1.0^{\mathrm{c} 2}$ & $24.3 \pm 0.9^{\mathrm{d} \#}$ \\
\hline LD 368 & $273.1 \pm 2.8^{\mathrm{a} 1}$ & $25.1 \pm 1.0^{\mathrm{b}^{*}}$ & $176.5 \pm 1.7^{\mathrm{c} 2}$ & $26.6 \pm 1.0^{\mathrm{d} \#}$ \\
\hline
\end{tabular}

Means in the same row followed by different digits (fertilized bran: fertilized kernel) /letters (fertilized bran: fertilized krenel)/symbols (fertilized kernel:non fertilized kernel) are significantly different at $95 \%$ confidence level $(\mathrm{p}>0.05)$ 


\section{Nutritional significance of minerals}

$\mathrm{Ca}$ is an important mineral for the synthesis of skeletal functions. $\mathrm{Mg}$ is a significant facilitator for many of the biochemical functions. $\mathrm{Mn}$ and $\mathrm{Zn}$ which are identified as trace minerals are important for many of the physiological functions.

Table 2. Mean calcium, and magnesium, contents of rice varieties with white pericarp $(\mathrm{mg} / \mathrm{kg})$

\begin{tabular}{|c|c|c|c|c|}
\hline & \multicolumn{4}{|c|}{ Calcium } \\
\hline & \multicolumn{2}{|c|}{ Fertilized } & \multicolumn{2}{|c|}{ Non- fertilized } \\
\hline & Bran & Kernel & Bran & Kernel \\
\hline BG 450 & $1296.0 \pm 29.6^{\mathrm{a} 1}$ & $772.5 \pm 10.0^{\mathrm{b}^{*}}$ & $675.3 \pm 14.1^{\mathrm{c} 2}$ & $620.4 \pm 2.5^{\mathrm{c \#}}$ \\
\hline BG400-1 & $1325.6 \pm 32.9^{\mathrm{a} 1}$ & $670.5 \pm 17.5^{\mathrm{b}^{*}}$ & $552.4 \pm 5.3^{\mathrm{c} 2}$ & $167.8 \pm 50.1^{\mathrm{d} \#}$ \\
\hline BG 360 & $1467.8 \pm 4.9^{\mathrm{a} 1}$ & $693.7 \pm 13.2^{\mathrm{b}^{*}}$ & $593.8 \pm 8.0^{\mathrm{c} 2}$ & $760.5 \pm 0.7^{\mathrm{d} \#}$ \\
\hline BG 94-1 & $1380.7 \pm 17.0^{\mathrm{a} 1}$ & $684.4 \pm 5.1^{\mathrm{b}^{*}}$ & $492.2 \pm 7.9^{\mathrm{c} 2}$ & $522.9 \pm 10.6^{\mathrm{d} \#}$ \\
\hline BG 379-2 & $1442.8 \pm 5.5^{\mathrm{a} 1}$ & $734.3 \pm 5.5^{\mathrm{b}^{*}}$ & $1251.5 \pm 11.2^{\mathrm{c} 2}$ & $659.5 \pm 14.7^{\mathrm{d} \#}$ \\
\hline BG 300 & $1456.2 \pm 24.6^{\mathrm{a} 1}$ & $687.0 \pm 0.4^{b^{*}}$ & $1345.1 \pm 2.0^{c 2}$ & $629.2 \pm 6.4^{\mathrm{d} \#}$ \\
\hline BG 305 & $1510.8 \pm 6.2^{\mathrm{a} 1}$ & $829.2 \pm 3.4^{\mathrm{b}^{*}}$ & $1281.1 \pm 88.2^{\mathrm{c} 2}$ & $501.9 \pm 0.9^{\mathrm{d} \#}$ \\
\hline BG 357 & $1437.6 \pm 12.2^{\mathrm{a} 1}$ & $693.1 \pm 8.5^{\mathrm{b}^{*}}$ & $1291.6 \pm 17.8^{\mathrm{c} 2}$ & $655.2 \pm 10.4^{\mathrm{d} \#}$ \\
\hline BW 367 & $1476.0 \pm 6.7^{\mathrm{a} 1}$ & $671.0 \pm 13.9^{\mathrm{b}^{*}}$ & $1309.7 \pm 4.1^{\mathrm{c} 2}$ & $618.4 \pm 16.3^{\mathrm{d} \#}$ \\
\hline BW 451 & $1162.1 \pm 14.8^{\mathrm{a} 1}$ & $643.0 \pm 7.1^{\mathrm{b}^{*}}$ & $1445.3 \pm 9.7^{\mathrm{c} 2}$ & $603.9 \pm 6.5^{\mathrm{d} \#}$ \\
\hline LD 371 & $1345.5 \pm 10.6^{\mathrm{a} 1}$ & $709.8 \pm 10.3^{\mathrm{b}^{*}}$ & $1202.0 \pm 8.5^{\mathrm{c} 2}$ & $682.1 \pm 8.4^{\mathrm{d} \#}$ \\
\hline AT 306 & $1254.2 \pm 30.3^{\mathrm{a} 1}$ & $537.0 \pm 6.3^{\mathrm{b}^{*}}$ & $1441.3 \pm 26.3^{\mathrm{c} 2}$ & $761.4 \pm 6.3^{\mathrm{d} \#}$ \\
\hline AT 309 & $1151.6 \pm 31.3^{\mathrm{a} 1}$ & $631.1 \pm 5.7^{\mathrm{b}^{*}}$ & $1418.4 \pm 16.5^{\mathrm{c} 2}$ & $892.0 \pm 9.6^{\mathrm{d} \#}$ \\
\hline \multirow[t]{2}{*}{ AT 405} & $952.5 \pm 20.6^{\mathrm{a} 1}$ & $616.4 \pm 5.8^{\mathrm{b}^{*}}$ & $1319.9 \pm 20.9^{\mathrm{c} 2}$ & $806.9 \pm 8.8^{\mathrm{d} \#}$ \\
\hline & \multicolumn{4}{|c|}{ Magnesium } \\
\hline BG 450 & $1174.3 \pm 13.3^{\mathrm{a} 1}$ & $373.1 \pm 3.8^{\mathrm{b}^{*}}$ & $1158.2 \pm 1.9^{\mathrm{c} 2}$ & $384.8 \pm 2.5^{\mathrm{d} \#}$ \\
\hline BG400-1 & $1173.1 \pm 7.4^{\mathrm{a} 1}$ & $227.8 \pm 1.9^{\mathrm{b}^{*}}$ & $1117.7 \pm 11.3^{\mathrm{c} 2}$ & $227.4 \pm 1.3^{\mathrm{d} \#}$ \\
\hline BG 360 & $1152.0 \pm 6.8^{\mathrm{a} 1}$ & $285.0 \pm 2.4^{\mathrm{b}^{*}}$ & $1128.5 \pm 12.8^{\mathrm{c} 2}$ & $366.7 \pm 1.5^{\mathrm{d} \#}$ \\
\hline BG 94-1 & $1178.0 \pm 0.6^{\mathrm{a} 1}$ & $193.3 \pm 2.4^{\mathrm{b}^{*}}$ & $1144.0 \pm 6.2^{\mathrm{c} 2}$ & $211.7 \pm 0.4^{\mathrm{d} \#}$ \\
\hline BG 379-2 & $1144.6 \pm 6.5^{\mathrm{a} 1}$ & $276.5 \pm 3.5^{\mathrm{b}^{*}}$ & $1172.3 \pm 1.7^{\mathrm{c} 2}$ & $241.7 \pm 3.5^{\mathrm{d} \#}$ \\
\hline BG 300 & $1124.3 \pm 11.1^{\mathrm{a} 1}$ & $250.0 \pm 0.9^{b^{*}}$ & $1129.6 \pm 11.6^{\mathrm{a} 2}$ & $338.2 \pm 1.9^{\mathrm{b \#}}$ \\
\hline BG 305 & $1166.2 \pm 6.9^{\mathrm{a} 1}$ & $272.3 \pm 3.0^{\mathrm{b}^{*}}$ & $1118.4 \pm 12.0^{\mathrm{c} 2}$ & $217.9 \pm 0.4^{\mathrm{d} \#}$ \\
\hline BG 357 & $1152.2 \pm 0.5^{\mathrm{a} 1}$ & $230.3 \pm 1.9^{b^{*}}$ & $1146.6 \pm 0.7^{\mathrm{a} 2}$ & $269.2 \pm 1.1^{\mathrm{b} \#}$ \\
\hline BW 367 & $1127.1 \pm 0.9^{\mathrm{a} 1}$ & $313.8 \pm 1.8^{\mathrm{b}^{*}}$ & $1136.9 \pm 11.8^{\mathrm{a} 2}$ & $296.5 \pm 0.7^{\mathrm{b \#}}$ \\
\hline BW 451 & $1184.2 \pm 11.4^{\mathrm{a} 1}$ & $487.3 \pm 3.7^{\mathrm{b}^{*}}$ & $1095.1 \pm 13.1^{\mathrm{c} 2}$ & $254.5 \pm 0.2^{\mathrm{d} \#}$ \\
\hline LD 371 & $1184.1 \pm 6.3^{\mathrm{a} 1}$ & $353.6 \pm 2.2^{\mathrm{b}^{*}}$ & $1068.2 \pm 6.5^{\mathrm{c} 2}$ & $257.2 \pm 2.2^{\mathrm{d} \#}$ \\
\hline AT 306 & $1205.7 \pm 13.8^{\mathrm{a} 1}$ & $286.7 \pm 1.5^{\mathrm{b}^{*}}$ & $1142.7 \pm 7.2^{\mathrm{c} 2}$ & $285.0 \pm 2.6^{\mathrm{d} \#}$ \\
\hline AT 309 & $1229.6 \pm 8.6^{\mathrm{a} 1}$ & $453.0 \pm 3.9^{\mathrm{b}^{*}}$ & $1192.6 \pm 0.7^{\mathrm{c} 2}$ & $349.2 \pm 1.8^{\mathrm{d} \#}$ \\
\hline AT 405 & $1132.6 \pm 13.2^{\mathrm{a} 1}$ & $262.6 \pm 1.4^{\mathrm{b}^{*}}$ & $1147.4 \pm 11.4^{\mathrm{a} 2}$ & $348.4 \pm 2.0^{\mathrm{d} \#}$ \\
\hline
\end{tabular}

Means in the same row followed by different digits(fertilized bran: fertilized kernel) /letters(fertilized bran: fertilized krenel)/symbols(fertilized kernel:non fertilized kernel) are significantly different at $95 \%$ confidence level. $(p>0.05)$ 
The per capita availability of rice is $100 \mathrm{Kg}$ /year. Accordingly the contribution of fertilization to the $\mathrm{Ca}, \mathrm{Mg}, \mathrm{Zn}$ content $(168.5-304 \mathrm{mg}),(61.6-124 \mathrm{mg}),(4.12-7.15 \mathrm{mg})$, respectively is below the RDA value of the above minerals. The reported Mn content (505-7.4mg) is higher than the RDA values.

\section{Effect of processing on the mineral content of rice varieties}

Sarwar et al., (2009) reported a similar trend in the variations of $\mathrm{Ca}$ and $\mathrm{Mg}$ of husk and whole grain fractions of Pakistani rice variety with application of different levels of organic and inorganic fertilizers compared to control (Non-fertilized). They further reported that fertilized husk and grains showed higher $\mathrm{Ca}$ and $\mathrm{Mg}$ contents than that of control.

Table 3. Mean zinc and manganese contents of rice varieties with white pericarp $(\mathrm{mg} / \mathrm{kg})$

\begin{tabular}{|c|c|c|c|c|}
\hline & \multicolumn{4}{|c|}{ Zinc } \\
\hline & \multicolumn{2}{|c|}{ Fertilized } & \multicolumn{2}{|c|}{ Non-fertilized } \\
\hline & Bran & Kernel & Bran & Kernel \\
\hline BG 450 & $154.0 \pm 2.6^{\mathrm{a} 1}$ & $18.0 \pm 0.5^{b^{*}}$ & $127.6 \pm 0.6^{\mathrm{c} 2}$ & $23.7 \pm 0.4^{\mathrm{d} \#}$ \\
\hline BG400-1 & $146.4 \pm 0.8^{\mathrm{a} 1}$ & $21.5 \pm 0.4^{\mathrm{b}^{*}}$ & $140.7 \pm 0.9^{c 2}$ & $20.4 \pm 0.3^{\mathrm{d} \#}$ \\
\hline BG 360 & $168.0 \pm 0.8^{\mathrm{a} 1}$ & $17.8 \pm 0.3^{\mathrm{b}^{*}}$ & $106.7 \pm 0.2^{\mathrm{c} 2}$ & $26.4 \pm 0.3^{\mathrm{d} 3 \#}$ \\
\hline BG 94-1 & $159.9 \pm 1.1^{\mathrm{a} 1}$ & $17.0 \pm 0.4^{b^{*}}$ & $153.5 \pm 0.7^{\mathrm{c} 2}$ & $19.2 \pm 0.5^{\mathrm{d} \#}$ \\
\hline BG 379-2 & $139.7 \pm 1.3^{\mathrm{a} 1}$ & $18.8 \pm 0.0^{\mathrm{b}^{*}}$ & $156.4 \pm 0.9^{\mathrm{c} 2}$ & $17.7 \pm 0.0^{\mathrm{d} \#}$ \\
\hline BG 300 & $139.8 \pm 2.0^{\mathrm{a} 1}$ & $23.0 \pm 0.3^{b^{*}}$ & $197.5 \pm 0.8^{\mathrm{c} 2}$ & $23.0 \pm 0.5^{\mathrm{d} \#}$ \\
\hline BG 305 & $149.7 \pm 0.7^{\mathrm{a} 1}$ & $22.3 \pm 0.4^{\mathrm{b}^{*}}$ & $143.6 \pm 0.7^{\mathrm{c} 2}$ & $22.7 \pm 0.4^{\mathrm{d} \#}$ \\
\hline BG 357 & $155.0 \pm 1.7^{\mathrm{a} 1}$ & $17.9 \pm 0.4^{\mathrm{b}^{*}}$ & $114.9 \pm 0.6^{\mathrm{c} 2}$ & $18.3 \pm 0.2^{\mathrm{d} \#}$ \\
\hline BW 367 & $158.6 \pm 2.2^{\mathrm{a} 1}$ & $16.9 \pm 0.3^{b^{*}}$ & $134.8 \pm 2.0^{\mathrm{c} 2}$ & $20.0 \pm 0.1^{\mathrm{d} \#}$ \\
\hline BW 451 & $171.3 \pm 1.4^{\mathrm{a} 1}$ & $23.7 \pm 0.2^{\mathrm{b}^{*}}$ & $126.9 \pm 1.1^{\mathrm{c} 2}$ & $19.3 \pm 0.3^{\mathrm{d} \#}$ \\
\hline LD 371 & $132.3 \pm 1.7^{\mathrm{a} 1}$ & $23.1 \pm 0.2^{\mathrm{b}^{*}}$ & $116.6 \pm 1.4^{\mathrm{c} 2}$ & $25.5 \pm 0.2^{\mathrm{d} \#}$ \\
\hline AT 306 & $167.3 \pm 1.4^{\mathrm{a} 1}$ & $25.3 \pm 0.5^{\mathrm{b}^{*}}$ & $190.7 \pm 2.3^{\mathrm{c} 2}$ & $31.6 \pm 0.4^{\mathrm{d} \#}$ \\
\hline AT 309 & $146.5 \pm 0.7^{\mathrm{a} 1}$ & $21.9 \pm 0.1^{\mathrm{b}^{*}}$ & $210.5 \pm 2.4^{\mathrm{c} 2}$ & $32.9 \pm 0.3^{\mathrm{d} \#}$ \\
\hline \multirow[t]{2}{*}{ AT 405} & $166.1 \pm 1.9^{\mathrm{a} 1}$ & $25.2 \pm 0.5^{b^{*}}$ & $126.0 \pm 2.2^{\mathrm{c} 2}$ & $25.4 \pm 0.1^{\mathrm{d} \#}$ \\
\hline & \multicolumn{4}{|c|}{ Manganese } \\
\hline BG 450 & $184.3 \pm 3.2^{\mathrm{a} 1}$ & $25.3 \pm 0.5^{\mathrm{b}^{*}}$ & $175.4 \pm 1.7^{\mathrm{a} 1}$ & $24.3 \pm 0.4^{\mathrm{cH}}$ \\
\hline BG400-1 & $142.0 \pm 1.5^{\mathrm{a} 1}$ & $20.5 \pm 0.7^{b^{*}}$ & $94.8 \pm 0.5^{\mathrm{c} 2}$ & $21.6 \pm 1.6^{\mathrm{d} \#}$ \\
\hline BG 360 & $214.3 \pm 3.3^{\mathrm{a} 1}$ & $25.3 \pm 1.5^{\mathrm{b}^{*}}$ & $98.2 \pm 1.7^{\mathrm{c} 2}$ & $23.0 \pm 0.5^{\mathrm{d} \#}$ \\
\hline BG 94-1 & $150.6 \pm 2.4^{\mathrm{a} 1}$ & $21.3 \pm 0.5^{\mathrm{b}^{*}}$ & $109.8 \pm 0.6^{\mathrm{c} 2}$ & $19.9 \pm 1.3^{\mathrm{d} \#}$ \\
\hline BG 379-2 & $188.1 \pm 1.1^{\mathrm{a} 1}$ & $34.5 \pm 0.4^{b^{*}}$ & $85.8 \pm 0.6^{\mathrm{c} 2}$ & $25.7 \pm 0.6^{\mathrm{d} \#}$ \\
\hline BG 300 & $151.4 \pm 1.5^{\mathrm{a} 1}$ & $24.0 \pm 1.1^{\mathrm{b}^{*}}$ & $126.5 \pm 1.3^{\mathrm{c} 2}$ & $20.8 \pm 0.6^{\mathrm{d} \#}$ \\
\hline BG 305 & $130.6 \pm 0.8^{\mathrm{a} 1}$ & $23.6 \pm 1.0^{\mathrm{b}^{*}}$ & $119.5 \pm 1.2^{\mathrm{a} 1}$ & $18.7 \pm 0.8^{\mathrm{d} \#}$ \\
\hline BG 357 & $131.9 \pm 0.6^{\mathrm{a} 1}$ & $20.8 \pm 0.6^{b^{*}}$ & $102.5 \pm 1.0^{\mathrm{c} 1}$ & $22.5 \pm 1.3^{\mathrm{d} \#}$ \\
\hline BW 367 & $151.3 \pm 0.9^{\mathrm{a} 1}$ & $20.7 \pm 1.1^{\mathrm{b}^{*}}$ & $158.5 \pm 1.4^{\mathrm{a} 2}$ & $23.9 \pm 0.7^{\mathrm{d} \#}$ \\
\hline BW 451 & $182.0 \pm 1.0^{\mathrm{a} 1}$ & $25.9 \pm 1.8^{\mathrm{b}^{*}}$ & $128.7 \pm 0.6^{\mathrm{c} 2}$ & $20.9 \pm 0.3^{\mathrm{d} \#}$ \\
\hline LD 371 & $164.1 \pm 0.2^{\mathrm{a} 1}$ & $25.9 \pm 0.4^{\mathrm{b}^{*}}$ & $142.5 \pm 1.4^{\mathrm{a} 1}$ & $24.8 \pm 0.2^{\mathrm{d} \#}$ \\
\hline AT 306 & $193.7 \pm 1.8^{\mathrm{a} 1}$ & $22.8 \pm 0.2^{\mathrm{b}^{*}}$ & $130.5 \pm 1.5^{\mathrm{c} 2}$ & $19.4 \pm 1.1^{\mathrm{d} \#}$ \\
\hline AT 309 & $169.6 \pm 1.9^{\mathrm{a} 1}$ & $23.6 \pm 0.2^{\mathrm{b}^{*}}$ & $142.3 \pm 1.4^{\mathrm{c} 2}$ & $20.3 \pm 0.5^{\mathrm{d} \#}$ \\
\hline AT 405 & $210.7 \pm 0.3^{\mathrm{a} 1}$ & $27.4 \pm 0.4^{\mathrm{b}^{*}}$ & $141.2 \pm 1.9^{\mathrm{c} 2}$ & $23.3 \pm 0.2^{\mathrm{d} \#}$ \\
\hline
\end{tabular}

Means in the same row followed by different digits(fertilized bran: fertilized kernel) /letters(fertilized bran: fertilized krenel)/symbols(fertilized kernel:non fertilized kernel) are significantly different at $95 \%$ confidence level. $(\mathrm{p}>0.05)$ 
The Ca and Mg contents of control husk (Non fertilized) were $2007 \mathrm{mg} / \mathrm{kg}$ and $401 \mathrm{mg} / \mathrm{kg}$, respectively, whereas inorganically fertilized husk was reported $3477 \mathrm{mg} / \mathrm{kg}$ and $530 \mathrm{mg} / \mathrm{kg}$ of $\mathrm{Ca}$ and $\mathrm{Mg}$, respectively. $\mathrm{Ca}$ and $\mathrm{Mg}$ contents in control rice grains were $778 \mathrm{mg} / \mathrm{kg}$ and $255 \mathrm{mg} / \mathrm{kg}$, respectively, and inorganically fertilized rice grains showed $1041 \mathrm{mg} / \mathrm{kg}$ and $355 \mathrm{mg} / \mathrm{kg}$ of $\mathrm{Ca}$ and $\mathrm{Mg}$, respectively. This supported the findings of the present study that the application of inorganic fertilizers strengthened the mineral content of rice grains and husk.

Recently, Verma and Srivastav (2017) showed that the mineral contents of polished counterparts of some aromatic and non-aromatic rice varieties grown in India. Their results showed that $\mathrm{Ca}, \mathrm{Mg}$ and $\mathrm{Zn}$ contents of rice ranged $63-99 \mathrm{mg} / \mathrm{kg}, 83-182 \mathrm{mg} / \mathrm{kg}$ and $9-17$ $\mathrm{mg} / \mathrm{kg}$, respectively. The lower levels of $\mathrm{Ca}$ and $\mathrm{Mg}$ obtained in their study compared to the present study could be due to the variations in geographical locations, soil properties like $\mathrm{pH}$, cation exchange capacity and leaching level of minerals, fertilization rate and techniques and the plant properties to absorb certain minerals (Leigh and Wyn Jones, 1984). The degree of milling which removes the most of the micronutrients severely affects the mineral composition. Processing operations of rice, namely dehulling, milling, and polishing affect the mineral contents. The mineral content variations among $100 \%$ rough rice, $82 \%$ brown rice and $72 \%$ milling rice. The Ca contents reported were 300,100 and $100 \mathrm{mg} / \mathrm{kg}$ for $100 \%$ rough rice, $82 \%$ brown rice and $72 \%$ milling rice, respectively (Abbas et al., 2011). In addition, Wang and coworkers (2011) demonstrated the variations of mineral content between bran and kernel fractions of three Indica rice cultivars. The ranges of $\mathrm{Ca}, \mathrm{Zn}, \mathrm{Mn}$ contents of bran were 682 $1331,38-56$ and 160-232 mg/kg, respectively. Further the ranges of 52-76, 19-29 and 10-28 $\mathrm{mg} / \mathrm{kg}$ of $\mathrm{Ca}, \mathrm{Zn}$ and $\mathrm{Mn}$, respectively were reported for kernel fractions. The trend of variations revealed in the present work tallied with the previous study by Wang et al., (2011). Mineral contents of the used rice varieties were significantly affected by variety, fertilization and processing $(P<0.05)$. In addition, the interactive effects of variety and fertilization, variety and polishing, fertilization and processing and variety, fertilization and processing also showed a significant effect on the mineral composition of selected rice varieties $(P<0.05)$. There are limited studies on the mineral content of newly improved Sri Lankan rice varieties. Further research are warranted to validate the results obtained in this study.

\section{CONCLUSIONS}

The application of inorganic fertilizers strengthen the mineral contents $(\mathrm{Ca}, \mathrm{Mg}, \mathrm{Mn}$, and $\mathrm{Zn}$ ) of rice kernels and brans of selected Sri Lankan newly improved rice varieties.

\section{ACKNOWLEDGEMENT}

This research was supported by the Wayamba University Research Grant Scheme (SRHDC/RP/04/15-20) through a research grant to AC. Dr Gamika Prathapasinghe was acknowledged by authors for the support extended for the mineral analysis. 


\section{REFERENCES}

Abbas, A., Murtaza, S., Faiza, A. K. and Naheed, S. R., S. (2011). Effect of processing on nutritional value of rice (Oryza Sativa). World Med Sci, 6(2), 68-73.

Anjum, F.M., Pasha, I., Bugti, M.A. and Butt, M.S. (2007). Mineral sciences composition of different rice varieties snd their milling fractions. Pakistan J Agric, 44(2), 332-336.

Diyabalanage, S., Navarathna, T., Abeysundara, H.T.K., Rajapakse, S. and Chandrajith, R. (2016). Trace elements in native and improved paddy rice from different climatic regions of Sri Lanka: implications for public health. Springer Plus, 5, 1864.

Ekanayake, H. (2009). The impact of fertilizer subsidy on paddy cultivation in Sri Lanka. Staff studies 3, Pp 74-96.

Oko, A.O., Ubi, B.E., Efisue, A.A. and Dambaba, N. (2012). Comparative analysis of the chemical nutrient composition of selected local and newly introduced rice varieties grown in Ebonyi State of Nigeria. Inter J Agric Forestry, 2(2), 16-23.

Leigh R.A., and Wyn Jones, R.G. (1984). Hypothesis relating critical potassium concentrations for growth to the distribution and functions of this ion in the plant cell. New Phytologist 97, 1-13.

Sarwar, G., Schmeisky, H., Hussain, N., Muhammad, S., Tahir, A., and Saleem, U. (2009). Variations in nutrient concentrations of wheat and paddy as affected by different levels of compost and chemical fertilizer in normal soil. Pakistan J Bot 41(5), 2403-2410.

Verma, D.K. and Srivastav, P.P. (2017). Proximate composition, mineral content and fatty acids analyses of aromatic and non-aromatic Indian rice [Online]. Rice Science, 24(1), 21-31.

Wang, K. M., Wu, J. G., Li, G., Zhang, D. P., Yang, Z. W., and Shi, C. H.(2011).Distribution of phytic acid and mineral elements in three indica rice (Oryza sativa L.) cultivars. J Cereal Sci, 54(1), 116-121. 